\title{
MODELO DE UN SISTEMA MULTI-AGENTE PARA LA OPTIMIZACIÓN DE LA CADENA DE SUMINISTROS EN LA INDUSTRIA DE LA MADERA DE CONÍFERAS
}

\section{MUTI-AGENT SYSTEM MODEL FOR THE OPTIMIZATION OF SOFTWOOD INDUSTRY SUPPLY CHAIN}

\author{
Mario Ramos ${ }^{1}$, Thomas Maness ${ }^{2}$, David Salinas ${ }^{3,4}$
}

\begin{abstract}
RESUMEN
El sector industrial maderero forma una cadena productiva, resumida en tres procesos: Aserrío, Secado y Remanufactura. Satisfacer el volumen demandado y cumplir con las fechas de entrega de los pedidos es importante tanto, para la cadena de producción, como para el cliente. La no integración de estos procesos, genera problemas de coherencia en los planes de producción, por lo que un modelo integrado del sector forestal, utilizando las ventajas de funcionamiento de los sistemas multi-agentes, serviría para mejorar la coherencia y el rendimiento global del sistema. El modelo de integración presentado en este trabajo, minimiza la tardanza global del sector industrial maderero y la comunicación entre los agentes, se realiza mediante la utilización de Contract Net Protocol. Los resultados muestran que en el aserradero se genera un mayor atraso para el sistema y es el cuello de botella en el cumplimiento de las órdenes de producción. Los resultados indicaron que se produce un atraso de entre 2 a 4 días en el cumplimiento de las órdenes de producción en la cadena, con un máximo de 20 días.
\end{abstract}

Palabras Clave: Industria de la madera, metaheurística, negociación, Pinus radiata, tardanza.

\begin{abstract}
The lumber industry sector forms a productive chain that be summarized in the three processes: sawing, drying and remanufacturing. Where satisfying the requested volume and meet the planned delivery dates is important to both, the client and each production chain. The non-integration presents coherence issues in the production plans. Hence, an integrated model in lumber sector, that uses the working advantages of multi-agents systems, works to improve coherence and the system's global productivity. The integration model presented in this paper minimizes global tardiness of the lumber transformation chain, at the moment of delivering production orders. Within the system, the communication between agents is done by using the Contract Net Protocol. The results show the sawmill, as a first step in the system, is where the biggest tardiness and bottle neck in the fulfilling of the production orders is produced. In average, the results showed a tardiness between 2 to 4 days in the compliance of chain production orders. The maximum delay is of 20 days.
\end{abstract}

Keywords: Lumber industry, metaheuristic, negotiation, Pinus radiata, tardiness.

\footnotetext{
${ }^{1}$ Profesor asociado, Universidad del Bío-Bío, Concepcion, Chile.

${ }^{2}$ Profesor titular, Oregón State University, Corvallis, USA.

${ }^{3}$ Alumno de Doctorado en Ciencias e Industrias de la Madera, Universidad del Bío-Bío, Concepcion, Chile.

^Autor para correspondencia: david.salinas.s@usach.cl

Recibido: 19.05.2014 Aceptado: 22.02.2015
} 


\section{INTRODUCCIÓN}

Las empresas productivas se enfrentan con diversos problemas al momento de la programar y planificar la producción. Los niveles de inventario, la disponibilidad de recursos, la cooperación y negociación entre empresas, nivel de ganancias y fechas de entrega de los productos son algunos de estos problemas, y es el planificador quien debe solucionar estos problemas, mediante la utilización de diferentes herramientas.

Dentro de los problemas antes mencionados, cumplir con la fecha de entrega de los productos terminados representa una problemática económica y de imagen corporativa frente a los clientes por lo que, la minimización de la tardanza en la cadena de producción es relevante a la hora de ser competitivos globalmente. Para una mayor cantidad de indicadores y factores de competitividad del sector forestal (Ponce et al. 2007).

Diversas investigaciones han destacado que el problema de minimizar la tardanza y lograr una correcta programación de la producción ya es difícil para una sola empresa por lo que, para la cadena completa de transformación de la madera, debería ser aún más complejo. De lo anterior, la planificación completa de transformación de la madera bajo este indicador, incrementa naturalmente la complejidad y solución del problema (Moyaux et al. 2006).

La planificación jerárquica, es el método de planificación que más se encuentra en la literatura para planificar la cadena de suministros (supply chain) sin embargo, al reunir una gran cantidad de variables en un sólo modelo centralizado, se vuelve rígido y la velocidad de respuesta se hace más lento (Frayret et al. 2004). Por una parte, los sistemas que son fuertemente centralizados y jerárquicos, representan un cuello de botella, el que hace difícil una reacción con la flexibilidad deseada, para las diversas fallas que ocurren al mismo tiempo y en condiciones de tiempo real (Hahndel et al. 1996). Los enfoques centralizados pueden ser adecuados dentro de un dominio de planificación local, sin embargo se consideran insuficientes para cubrir situaciones más complejas y realistas.

Para superar lo anterior, un conjunto de enfoques han sido desarrollados y probados, especialmente los basados en la investigación sobre sistemas multi-agentes, los que han demostrado un gran potencial. Este es el caso de los modelos descentralizados que producen planes distribuidos y, están coordinados por agentes que actúan en colaboración para formar un sistema planificación (Santa Eulalia et al. 2007).

Por otra parte, estos sistemas de producción y planificación, cuentan con equipos procesadores de datos, habilidades de memorización y comunicación que permiten autonomía en la toma de decisiones. La idea, no es solamente reaccionar rápido a situaciones internas o externas al sistema sino que también cumplir con los objetivos de producción planteados (Charpentier y Muhl 2004). Cada socio de la cadena será un agente que colaborará y negociará con los otros para obtener tanto ganancia a nivel local como en la cadena completa. Para Moyaux et al. (2003), el Efecto Látigo presente en la cadena de transformación de la madera, ubicado entre el aserradero, las bodegas de productos terminados y las ventas de los mismos, se produce por malas decisiones hechas en forma local, afectando al aserradero como primer eslabón productivo de la cadena. Para solucionar el problema anterior, se aplica y aprovechan las características distributivas y las fluctuaciones de un sistema multi-agentes, en un sistema de producción con constantes fluctuaciones en las demandas.

Generalizando, se pueden identificar en las empresas que componen la industria forestal distintos ambientes de producción. Tal como se aprecia en la Figura 1, el aserradero de la Etapa 1, puede considerarse como un ambiente de producción equivalente a "una" máquina en la cual el problema consiste en definir el orden de entrada a la máquina y los planes de corte para cortar la materia prima, en piezas de madera dimensionada. Maness y Adams (1991), utilizan el enfoque de máquina única en el aserradero y determinan esquemas de corte para una demanda agregada. 
FLUJO DE INFORMACIÓN

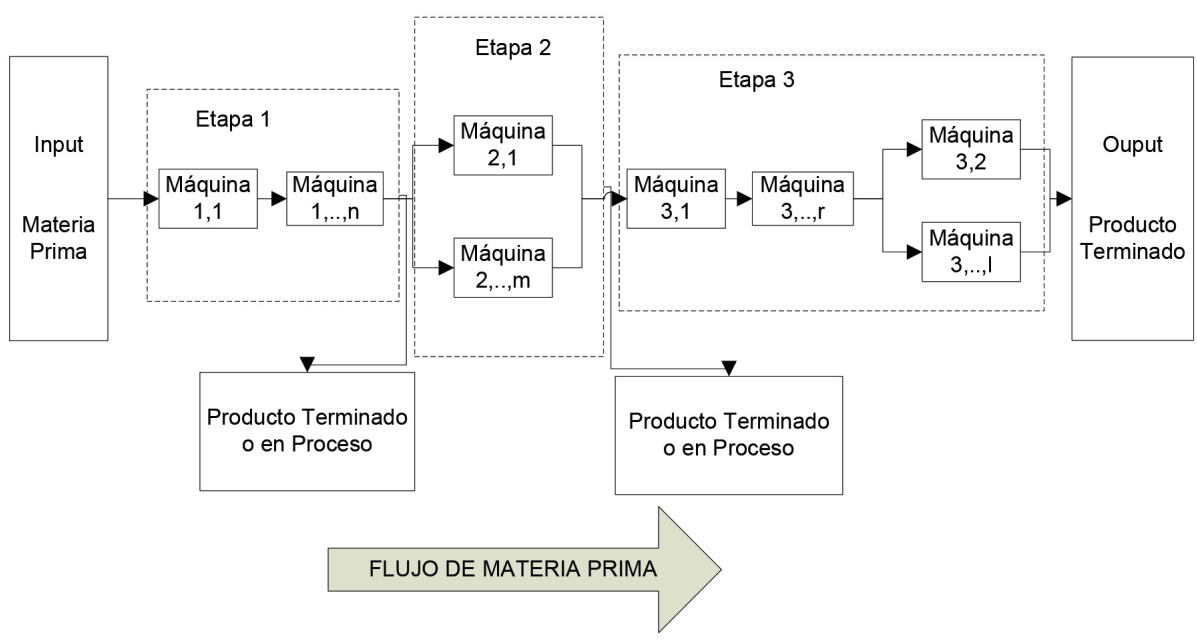

Figura 1. Ambientes de producción en la industria forestal.

(Fuente: Elaboración propia).

La Etapa 2, el secado, muestra un ambiente de producción de máquinas en paralelo, donde diferentes lotes de madera provenientes del proceso de aserrío, se procesan en diferentes secadores. En Gaudreault et al. (2006), se propone un modelo que integra el área de secado y el área de terminación (cepillado de la madera), utilizando un enfoque de sistemas multi-agentes para mejorar la programación de las cámaras de secado. Por último, en la Etapa 3 Se pueden encontrar alternadamente, ambientes tipo máquina única y máquinas en paralelo (Baesler et al. 2006). En Farrell y Mannes (2004), se propone un sistema de ayuda en la toma de decisiones en una planta de remanufactura. La investigación utiliza programación lineal, y aunque la aplicación desarrollada es específica para una planta en particular, resultó ser lo bastante flexible para ser utilizado en otras empresas de similares características.

Hasta el momento, se ha señalado la resolución de los problemas de producción, asociados a cada agente, de manera separada y la integración de las plantas parece a primera vista un error, pero por el contrario un modelo que asuma todos los requerimientos del conjunto en su modo de planificación, asegurará coherencia y favorecerá la obtención de un máximo global, el que difícilmente se alcance por separado. Para Singer y Donoso (2007), la industria maderera chilena, no está del todo integrada y las compañías encaran los problemas de cualquier cadena de suministro por lo que, un modelo integrado de ésta puede beneficiarlos.

Los modelos integrados de planificación de la producción son escasos. Charpentier y Muhl (2004), desarrollan un modelo de integración al interior de una planta de automóviles, integrando los tres procesos más importantes dentro de la línea de producción. Maness y Scott (2002), incorporan los valores de las piezas obtenidas en las plantas de elaboración terciaria, y analizan como afectan estos precios la producción de madera aserrada. En Gaudreault et al. (2006), se integra la planificación de la producción en los aserraderos y las plantas de secado, con el fin de disminuir la cantidad de órdenes devueltas por no cumplir con los plazos estipulados.

En un nivel de planificación operacional, incorporando el tiempo y la minimización de la tardanza como función global del sistema y, utilizando además el enfoque de los sistemas multi-agentes, no se han encontrado modelos de integración para la industria forestal. 
Como lo expresan Babayan y He (2004), los problemas que se presentan en la planificación de la producción son, generalmente, problemas que no tienen solución en tiempo polinomial (NP-hard), lo que hace improbable encontrar soluciones óptimas en tiempos razonablemente cortos. Por lo anterior, la utilización de heurísticas ofrecería un nuevo enfoque y buenas soluciones. En Mendoza et al. (1991), se utiliza un modelo de optimización para obtener la mejor combinación de trozos a procesar, y así cumplir con las órdenes de producción. Los autores reconocen que es difícil hacer un modelo general para toda la industria, debido a las diferencias inherentes que existen entre ellas, por lo que su modelo se refiere a una sola empresa. En Carino y Willis (2001), se propone un modelo de programación lineal de múltiples etapas que integra verticalmente la aplicación del patrón de corte en el proceso de aserrío, el secado y venta de madera. No incorpora los demás procesos productivos. En la misma línea, Maness y Scott (2002), plantean un modelo de planificación de la producción en multi periodos. El modelo permite determinar los costos de manejar altos niveles de inventario y determina estrategias de producción, con respecto a los patrones de corte y la característica de la madera a procesar. En Wessels et al. (2006), se presenta un modelo de integración entre la cosecha en el bosque y el proceso de aserrío, entregando como resultado los mejores patrones de corte que incrementan del ingreso.

Frayret et al. (2005), utiliza los sistemas multi-agentes y modela conceptualmente el proceso de conversión primaria. Se integra desde los trozos, como materias primas, hasta las ventas de madera seca y las etapas del proceso pueden colaborar entre sí. Se afirma que las decisiones tomadas tempranamente en los aserraderos, afectan la producción da la remanufactura. En Donald et al. (2001), se plantean dos maneras de ayudar a la producción en la industria secundaría. La primera es con modelo individual y la segunda, integrándola con la industria de transformación primaria. El modelo de integración incorpora el valor final de las piezas en la remanufactura, en la etapa previa del aserrío, influenciando la obtención de mejores patrones de corte, aplicados a los trozos que entran al aserradero. El modelo integrado obtiene un $10 \%$ más de ganancias económicas.

En Charpentier y Muhl (2004), se estudian la integración de una fábrica al hacer negociar entre sí las partes involucradas para obtener un máximo global del sistema. Se utilizan algoritmos genéticos como metaheurística y se introduce el concepto de Mérito Global presentado por Duffie y Prabhu (1994), para asegurar la convergencia del sistema.

Dudek y Stadtler (2005), plantean que un enfoque de planificación colaborativa y de negociación de manera centralizada, sería demasiado complejo a la hora de implementarlo. La coordinación "Aguas Arriba" entrega resultados que no son los mejores. Estos autores proponen un modelo de negociación entre proveedor y comprador basado en contratos, programación matemática y soluciones basadas en agentes.

Ribas-Vila y Companys (2006), plantean que en una cadena de producción formada por "N" socios y desde un punto de vista de una planificación centralizada, para lograr una optimización se debe considerar un único modelo que cubra las "N" etapas de planificación, con un modelo de planificación colaborativa que contribuya a mejorar la solución global.

Cid et al. (2007), plantean que las lógicas de producción "pull o push", son utilizadas como estrategias para gatillar la elaboración de productos, siendo la lógica pull la que involucra el flujo de información desde el mercado y produce decisiones aguas arriba, que tienden a mejorar las operaciones de producción. Según Frayret et al. (2005), está lógica de producción permite solucionar diferentes tipos de problemas implicados dentro de la cadena de suministro además de mejorar la logística interna de la empresa al contribuir a la reducción de ineficiencias del proceso (efecto látigo). 
Según, Singer y Donoso (2007), los investigadores han tratado de reunir varios tipos de decisiones en un modelo (mono o multi-criterio) o han tratado de reunir distintos requerimientos en una aplicación, buscando que esta funcione de manera ajustada a la realidad. Encontrado que la integración de diversos objetivos y restricciones ayuda a mejorar los distintos índices de medición de un modelo, siendo los sistemas multiagentes una buena alternativa para poder realizar una integración de distintas plantas. Huhns y Stephens (1999) plantean que generalmente los sistemas multi-agentes son sistemas menos eficientes que los sistemas centralizados, pero por el contrario, son más fáciles de aplicar y de entender cuando el problema al que son aplicados es distribuido. En la misma línea, Jenning (2000) plantea que la flexibilidad y los altos niveles de interacción de los agentes, hace que sistemas complejos sean fácilmente entendidos.

En este trabajo se propone un modelo de negociación y colaboración entre los socios de una cadena de producción, con el fin de cumplir con el objetivo de minimizar la tardanza como función global del sistema. La distribución de tareas específica en la planificación, desarrolladas por agentes autónomos y especializados (cada uno de los tres agentes socios del sistema: agente aserradero, agente secado, agente remanufactura), sin presencia de un agente superior y considerando una estructura distribuida (Frayret et al. 2004).

\section{MATERIALES Y MÉTODOS}

En la presente investigación se utilizó la lógica pull, empleando la información de los pedidos hechos desde el mercado y sus especificaciones en cuanto a volumen de productos y fechas de entrega de los pedidos. La información de la respuesta a la licitación de un producto se hizo mediante el Protocolo Contract Net, de la misma manera en que se realizó en Koller (2004):

1) El cliente al solicitar un determinado producto, desencadena la producción en el sistema, forzando a los agentes a comunicarse entre ellos para poder lograr lo solicitado. Se ha considerado para cada agente que al completar el volumen solicitado, se la función de minimización de la tardanza, sea la función objetivo de cada agente. Los agentes intercambian información con sus pares de forma trasparente, es decir, los datos transmitidos hacia un receptor son recibidos de forma íntegra sin ser modificados en el camino por alguna entidad. La operación interna de la red, entre agentes, se logra mediante el cumplimiento de dos requisitos: Todos los agentes usan el mismo lenguaje de comunicación y contenido. Lo anterior asegura que la información transmitida por el agente " $\mathrm{X}$ " pueda ser entendida por el agente "Y".

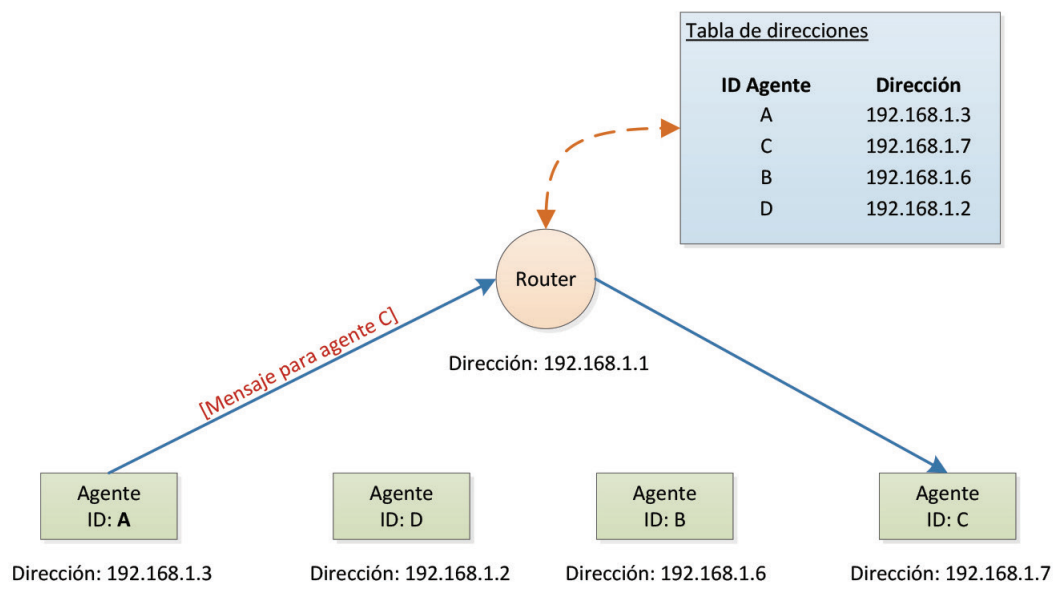

Figura 2. Comunicación entre agentes.

(Fuente: Elaboración propia). 
2) Todos los agentes forman parte de una misma red. La creación de una entidad "Router" permite la comunicación entre agentes, actuando como un servidor de red, donde se almacenan las direcciones y nombres de cada uno de los agentes que compone dicha red y desde donde se deriva la información recibida hacia el agente respectivo (Figura 2).

La entidad "Router" envía continuamente hacia el Agente Central los datagramas que se envían entre agentes durante la operación del sistema, monitoreando las negociaciones entre agentes y evitando que estas se extiendan de forma infinita. De esta forma el esquema general del sistema queda conformado como se muestra (Figura 3).

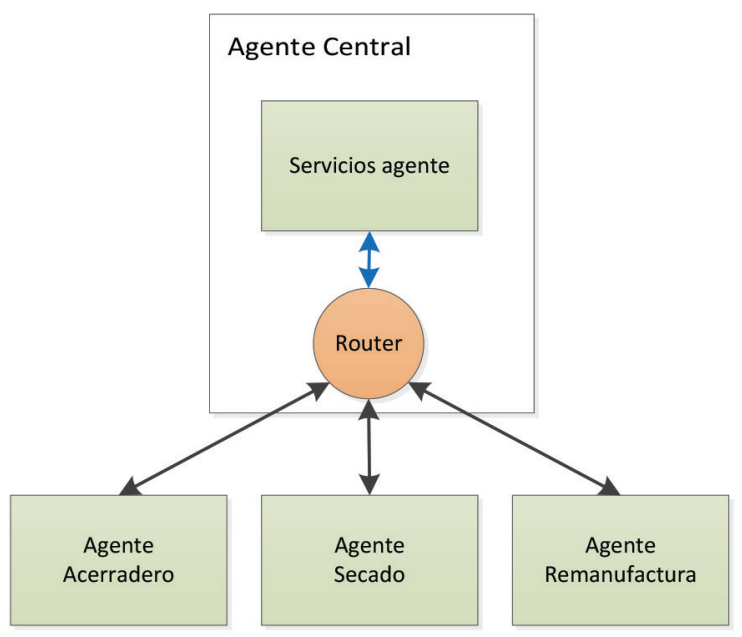

Figura 3. Integración de la cadena de transformación de la madera y ubicación agente central y Router. (Fuente: Elaboración propia).

Como puede apreciarse en la figura anterior, el componente "Router" maneja el flujo de información entre los agentes, permitiendo la comunicación entre estos. Su operación es a nivel de red y en ningún momento interviene en las decisiones de los agentes.

La estructura computacional, así como todos los componentes del modelo fueron implementados en IDE Borland C++ Builder 6, dado que este lenguaje permite la creación de aplicaciones, utilizando lenguaje de programación $\mathrm{C}++$, sobre una plataforma Windows XP SP3. La selección de este entorno se debe a las herramientas que dispone para realización de aplicaciones orientadas a objeto que permiten una arquitectura distribuida. Se utilizó también la base de datos My SQL Workbench 6.0 CE, por la posibilidad de interactuar directamente con Excel, facilitando el ingreso de los datos y la configuración de los parámetros del sistema.

El cliente puede enviar una orden de producción a cualquier agente del sistema, solicitando cualquiera de los productos terminados. Cada uno de los agentes evaluará, de acuerdo a su función objetivo, si es posible producir a tiempo y sin aumentar la tardanza del sistema. Una vez evaluada la situación y conociendo todos los tiempos que se demorará la orden de producción, se envía un mensaje al cliente. En la Figura 3, la interacción entre los agentes y la supervisión del sistema, se realiza mediante el monitoreo del proceso de negociación y los índices de tardanza de los agentes, durante cada negociación. El sistema evita que las negociaciones se estanquen y entren en un ciclo infinito. Cuando un estancamiento es detectado por el agente central, este envía un requerimiento al agente licitador para forzar la aceptación de la mejor propuesta que dispone. La supervisión del índice de tardanza global (IT Global) también se realiza de forma global y éste se determina como la sumatoria de los índices de tardanzas locales (IT Agente i) alcanzados por los agentes (1). 
Una vez que los tres agentes han reportado sus resultados locales, el agente global, determinará las posibles acciones a seguir y ordenará quien es el que debe mejorar en sus índices de producción. Este agente solamente entrará en el juego una vez que se produzcan conflictos, de no ser así, el agente sólo actuará para evaluar la función objetivo global.

Para asegurar la convergencia del sistema con el agente central envía mensajes a los agentes del sistema y cambia los parámetros de los pedidos para que los agentes vuelvan a iterar y forzarlos a encontrar mejores soluciones locales. El índice de Mérito Global (IT), también favorece la minimización de la tardanza global del sistema.

$$
I T_{\text {Global }}=\sum_{n} I T_{\text {Agente }_{n}}
$$

El registro del valor de los índices se realizó a partir de los datos obtenidos en el monitoreo de las actividades de negociación que fueron almacenados en una base de datos, consultable por el usuario. La última función realizada por el agente central permite al usuario ingresar sus pedidos al sistema y que éstos sean gestionados por el agente central.

Los escenarios de simulación propuestos, se adaptaron de la investigación realizada en Koller 2004 y se construyeron en base a pedidos ficticios, para los cuales se actualizaron las fechas de las entregas y se dejó igual los demás parámetros de los pedidos. Cada uno de estos escenarios cuenta con los siguientes datos de entrada al sistema: Un Código de Pedido (COD_PEDIDO) para diferenciar un pedido de otro y varía de 1 a 4. Una fecha de pedido (FECHA_PEDIDO) por escenario, para un periodo de un mes de producción. Esta fecha indica el ingresó al sistema y finalmente una fecha de entrega (ENTREGA).

Las fechas en las cuales las órdenes de producción ingresaron al sistema, se obtuvieron de manera aleatoria, utilizando la función RANDON, de Excel y la fecha de entrega se obtuvo utilizando un número aleatorio entre 1 y 30 (simulando un mes de producción). Utilizando la misma función RANDOM, se obtuvieron la cantidad de piezas de madera de cada pedido. Las dimensiones en milímetros, de las piezas solicitadas fueron: ancho $(65,90,100,110,125,125,130,150,160,200)$, espesor $(11,12,18,20,24,25)$ y largo $(3600,3800$, 4000), todas medidas reales.

Cada uno de estos escenarios se simuló un mínimo de 3 veces de manera independiente entre un escenario y otro. Entre cada simulación de un escenario, las piezas derivadas a bodega como inventario, se conservaron como un input para cada nueva corrida de la respectiva simulación. Una vez obtenidos los resultados de los tiempos de procesamientos y, por consiguiente, las fechas de entrega de los pedidos del cliente al agente aserradero, los resultados se traspasaron al agente secado, siguiente agente de la cadena de transformación de la madera. Una vez que los datos fueron procesados por el agente secado, estos fueron enviados al agente remanufactura, último agente del sistema. Todos los agentes utilizaron la regla de despacho EDD (fecha más temprana de entrega) como regla para minimizar la tardanza del sistema. 


\section{RESULTADOS Y DISCUSIÓN}

En esta investigación, los agentes trabajaron de forma manual y no automática. La integración de los resultados se produjo utilizando los resultados de cada salida de un agente, como los input del siguiente proceso. La Tabla 1, muestra los escenarios de simulación para el agente aserrío.

Tabla 1. Escenarios de simulación que ingresan al aserradero.

\begin{tabular}{|c|c|c|c|c|c|}
\hline \multicolumn{3}{|c|}{ Escenario 1 } & \multicolumn{3}{c|}{ Escenario 2 } \\
\hline COD PEDIDO & FECHA PEDIDO & ENTREGA & COD PEDIDO & FECHA PEDIDO & ENTREGA \\
\hline 1 & $01-12-2013$ & 7 & 1 & $01-12-2013$ & 15 \\
\hline 2 & $01-12-2013$ & 13 & 2 & $01-12-2013$ & 10 \\
\hline \multicolumn{7}{|c|}{ Escenario 3 } & & \multicolumn{3}{c|}{ Escenario 4 } \\
\hline COD PEDIDO & FECHA PEDIDO & ENTREGA & COD PEDIDO & FECHA PEDIDO & ENTREGA \\
\hline 1 & $01-12-2013$ & 14 & 1 & $12-12-2013$ & 6 \\
\hline 2 & $01-12-2013$ & 8 & 2 & $12-12-2013$ & 22 \\
\hline 3 & $01-12-2013$ & 18 & 3 & $12-12-2013$ & 11 \\
\hline \multicolumn{7}{|c|}{ Escenario 5 } & & & Escenario 6 & \\
\hline COD PEDIDO & FECHA PEDIDO & ENTREGA & COD PEDIDO & FECHA PEDIDO & ENTREGA \\
\hline 1 & $01-01-2014$ & 9 & 1 & $01-01-2014$ & 23 \\
\hline 2 & $01-01-2014$ & 17 & 2 & $01-01-2014$ & 12 \\
\hline 3 & $01-01-2014$ & 25 & 3 & $01-01-2014$ & 5 \\
\hline 4 & $01-01-2014$ & 13 & 4 & $01-01-2014$ & 17 \\
\hline
\end{tabular}

Los resultados indicaron que de los 18 escenarios (ESC), 8 resultaron sin atraso (valores negativos en Tabla 2) y los restantes 10 presentaron entre 1 a 16 días de atraso parcial, en la entrega de la órdenes de producción, (valores positivos en Tabla 2).

Las piezas de cada pedido, se asignan en cada uno de los trozos de madera, de acuerdo a lo planteado en el trabajo de Maness y Adams 1991, donde se busca maximizar el rendimiento volumétrico de cada una de las trozas, independizando a los aserraderos del uso de patrones de corte, preestablecidos.

En promedio los datos de la Tabla 2 indican que el atraso obtenido de las simulaciones, fue de 2,27 días. Además, los resultados indican que se obtuvo un atraso máximo de 16 días y un adelanto máximo de 9 días. Si para el pedido 1, la fecha de ingreso al sistema, se realizó el 01-12-2013 y se estipuló que la entrega de este pedido se realizara en 7 días, (fecha de entrega sería el 08-12-2013) los resultaron indican que este pedido se terminó de procesar el 24-12-2013, es decir con 16 días de atraso, tal como lo indica la tabla anterior. 
Tabla 2. Resultados del agente aserrío.

\begin{tabular}{|c|c|c|c|c|c|c|c|}
\hline ESC & $\begin{array}{c}\mathbf{N}^{\circ} \\
\text { PEDIDO }\end{array}$ & $\begin{array}{c}\text { FECHA_REAL } \\
\text { SALIDA }\end{array}$ & ATRASO & ESC & $\begin{array}{c}\mathbf{N}^{\circ} \\
\text { PEDIDO }\end{array}$ & $\begin{array}{c}\text { FECHA_REAL } \\
\text { SALIDA }\end{array}$ & ATRASO \\
\hline \multirow{2}{*}{1} & 1 & $24-12-2013$ & 16 & \multirow{2}{*}{2} & 1 & $19-12-2013$ & 3 \\
\hline & 2 & $11-12-2013$ & -3 & & 2 & 09-12-2013 & -2 \\
\hline \multirow{3}{*}{3} & 1 & $10-12-2013$ & -5 & \multirow{3}{*}{4} & 1 & 29-12-2013 & 11 \\
\hline & 2 & $12-12-2013$ & 3 & & 2 & $31-12-2013$ & -3 \\
\hline & 3 & $10-12-2013$ & -9 & & 3 & $27-12-2013$ & 4 \\
\hline \multirow{4}{*}{5} & 1 & 25-01-2014 & 1 & \multirow{4}{*}{6} & 1 & 22-01-2014 & -2 \\
\hline & 2 & 09-01-2014 & -4 & & 2 & $17-01-2014$ & 4 \\
\hline & 3 & 20-01-2014 & 14 & & 3 & $18-01-2014$ & 12 \\
\hline & 4 & $15-01-2014$ & -3 & & 4 & 22-01-2014 & 4 \\
\hline
\end{tabular}

Una vez obtenidos los resultados en cada escenario del aserradero, los datos resultantes se ingresaron al agente secado, considerando la fecha de salida del aserradero, Fecha_real_Salida, como la fecha en la cual la madera aserrada está disponible para ingresar al secador.

Los datos de entrada al agente secado, en cada uno de los escenarios de simulación, se ingresaron al sistema considerando las fechas de salida de las órdenes de producción del aserradero. Se constató además, la fecha en la que realmente la carga de madera ingresó al secador. La configuración de los parámetros del secado, en relación con los tiempos de secado, se estableció constante e igual a 72 horas por cada carga de secado.

En la tabla 3, se muestra una parte de los resultados obtenidos por el agente secado, al cargar los escenarios de simulación.

Tabla 3. Resultados agente secado.

\begin{tabular}{|c|c|c|c|c|c|c|c|c|c|c|c|}
\hline $\begin{array}{c}\text { COD_- } \\
\text { PED }\end{array}$ & E & A & L & T_MAD & CANT & F_ENT & F_DEM & $\begin{array}{c}\text { F_ENT_ } \\
\text { PROC }\end{array}$ & $\begin{array}{c}\text { F_SAL_ } \\
\text { PROC }\end{array}$ & GAP & ATRASO \\
\hline 1 & 20 & 90 & 380 & LATERAL & 288 & $24-12-2013$ & $25-12-2013$ & $\begin{array}{c}27-12-2013 \\
12: 00\end{array}$ & $\begin{array}{c}30-12-2013 \\
10: 00\end{array}$ & 3 & 5 \\
\hline 1 & 20 & 110 & 380 & LATERAL & 495 & $24-12-2013$ & $25-12-2013$ & $\begin{array}{c}27-12-2013 \\
12: 00\end{array}$ & $\begin{array}{c}30-12-2013 \\
10: 00\end{array}$ & 3 & 5 \\
\hline
\end{tabular}

Los resultados mostrados en la Tabla 3, indican que existe una diferencia entre la fecha en la que el paquete está listo para entrar al secador (F_ENT) y la fecha en la que realmente lo hace (F_ENT_PROC), esta diferencia se denomina GAP y los resultados muestran en promedio un GAP de 3 a 8 días. Una vez que ingresaron y fueron procesados los paquetes, los resultados indican un atraso de entre 2 a 4 días y un GAP máximo de 20 días. Finalmente, los resultados obtenidos a partir de la simulación indican que en promedio en el agente secado, se produjo un atraso negativo de -2,43 días, es decir los paquetes de madera salieron, antes de la fecha estipulada. Una vez que el proceso de secado terminó, las fechas de término del proceso se convirtieron en las fecha de ingreso al agente remanufactura. 
Tal como lo muestra la ¡Error! La autoreferencia al marcador no es válida., cada uno de los pedidos cuenta con un código de pedido (COD_PED), el tipo de moldura (TIPO_PAQ), cantidad de paquetes (CANT_PAQ) y el volumen solicitado de ese tipo de moldura (VOL), además de sus respectivas dimensiones de espesor (E), ancho (A) y largo (L). Se aprecia la fecha de entrada (F_ENT) al sistema (incluyendo la hora) y la fecha en la cual han sido finalizados los trabajos (F_SAL). De la misma manera que con los dos agentes anteriores, los trabajos en el agente remanufactura, se programaron de acuerdo a la orden de producción que tuviera la fecha de entrega más próxima.

En los resultados obtenidos, se aprecia que el agente remanufactura entregó todos sus pedidos a tiempo y no hubo pedidos con atraso. Cabe destacar, que el único atraso identificado se produjo debido a que un pedido fue programado antes de media noche, terminando su procesamiento un día después.

Tabla 4. Resultados agente remanufactura.

\begin{tabular}{|c|c|c|c|c|c|c|c|c|c|}
\hline $\begin{array}{c}\text { COD } \\
\text { PED }\end{array}$ & TIPO PAQ & $\begin{array}{c}\text { CANT } \\
\text { PAQ }\end{array}$ & VOL & E & A & L & F_ENT & F_SAL & ATRASO \\
\hline 1 & $\begin{array}{c}\text { MOLDURA } \\
\text { CARA }\end{array}$ & 3 & 0,0182628 & 0,02 & 0,09 & 3,8 & $\begin{array}{c}28-01-2014 \\
19: 02\end{array}$ & $\begin{array}{c}28-01-2014 \\
19: 06\end{array}$ & 0 \\
\hline 1 & $\begin{array}{c}\text { MOLDURA } \\
\text { CARA }\end{array}$ & 3 & 0,0182628 & 0,02 & 0,09 & 3,8 & $\begin{array}{c}28-01-2014 \\
19: 09\end{array}$ & $\begin{array}{c}28-01-2014 \\
19: 15\end{array}$ & 0 \\
\hline 1 & $\begin{array}{c}\text { MOLDURA } \\
\text { CARA }\end{array}$ & 3 & 0,0182628 & 0,02 & 0,09 & 3,8 & $\begin{array}{c}28-01-2014 \\
19: 18\end{array}$ & $\begin{array}{c}28-01-2014 \\
19: 24\end{array}$ & 0 \\
\hline 1 & $\begin{array}{c}\text { MOLDURA } \\
\text { CARA }\end{array}$ & 3 & 0,0182628 & 0,02 & 0,09 & 3,8 & $\begin{array}{c}28-01-2014 \\
19: 27\end{array}$ & $\begin{array}{c}28-01-2014 \\
19: 33\end{array}$ & 0 \\
\hline
\end{tabular}

\section{CONCLUSIONES}

Los resultados obtenidos muestran la pertinencia en la utilización de un sistema multi-agentes para integrar la cadena de transformación de la madera y, en utilizar como objetivo principal una función para minimizar el atraso en la elaboración de productos.

El modelo propuesto, bajo el ambiente de producción Flow Shop General, favorece la conceptualización del flujo de información y de materias primas a través del sistema, facilitando el desarrollo del sistema.

La utilización de la regla de despacho EDD en los agentes, ayudó a minimizar la tardanza de cada agente y del sistema.

Aunque el funcionamiento del sistema es manual, se aprecia que los tres agentes funcionan de manera articulada, aunque la velocidad de interacción entre el traspaso de los resultados de salida de un agente y la entrada como input en el agente siguiente, es una limitante en el sistema actual al no estar funcionando de manera automatizada.

El agente aserradero presenta un atraso máximo de 16 días en completar sus pedidos, siendo éste determinante en la demora en la entrega final de los productos. Una mejora en el orden de entrada de los pedidos en el aserradero mejoraría considerablemente el rendimiento del sistema.

El agente secado reporta un GAP máximo de 20 días y el administrar estas ventanas de tiempo, reduciría el tiempo total de entrega de todos los pedidos. 


\section{BIBLIOGRAFIA}

Babayan, A.; He, D. 2004. Solving the n-job 3-stage flexible flowshop scheduling problem using an agent-based approach. Int J Prod Res 42(4): 777-799.

Baesler, F.; Ceballos, L.; Ramírez, M. 2006. Programación Multiobjetivo de Máquinas Moldureras a través de algoritmos Meméticos. Maderas: Ciencia y tecnología 8(3):183-192.

Carino, H.; Willis, D. 2001. Enhancing the profitability of a vertically Integrated Wordproducts production system. Part 1 A multistage modelling approach. Forest Products Journal 51(4): 37-44.

Charpentier, P.; Muhl, E. 2004. From a reactive, heterachical to a holonic system: an application for optimizing flow in an automotive plant. Product Planning and Control 2: 166-177.

Cid, F.; Frayret, J.M.; Rousseau, A.; Léger, F. 2007. Evaluatión of pull strategies in lumber production planninig: A case study. $19^{\text {th }}$ International Conference on Production Research. Valparaíso. Chile.

Donald, S.; Maness, T.; Marinescu, M. 2001. Production planning for integrated primary and secondary lumber manufacturing. Wood and Fiber Science 33(3): 334-344.

Dudek, G.; Stadtler, H.. 2005. Negotiation-Based collaborative planning between supply chains partners. European Journal of Operational Research 163: 668-687.

Duffie, N. A.; Prabhu, V. V. 1994. Real-Time Distributed Scheduling of Heterarchical Manufacturing Systems. Journal of Manufacturing Systems 13(2): 94-107.

Farrell, R. R.; Maness T. C. 2004. A relational database approach to a linear programming-based decision support system for production planning in secondary wood product manufacturing. Decision Support Systems 40: 183-196.

Frayret, J.M.; D'Amours, S.; Montreuil, B. 2004. Coordination and control in distributed and agentbased manufacturing systems. Production Planning \& Control 15(1): 42- 54.

Frayret, J.M.; D'Amours, S.; Rousseau, A.; Harvey, S. 2005. Agent-based Supply Chain Planning in the forest products industry. Working Paper DT-2005-JMF-1. Research Consortium in e-Business in the Forest Products Industry(FOR@C), Network Organization Technology Research Center (CENTOR), Université Laval, Québec, Canada.

Gaudreault, J.; Frayret, J.M.; Rousseau, A.; D'Amours, S. 2006. Integrated planning and scheduling in a divergent production system with co production: a real-time perspectiva. Working Paper DT-2006JMF-1. Research Consortium in e-Business in the Forest Products Industry (FOR@C), Network Organization Technology Research Center (CENTOR), Université Laval, Québec, Canada.

Hahndel, S.; Fuchs, F.; Levi, P. 1996. Distributed Negotiation-based Task Planning for a Flexible Manufacturing Environment. Distributed Software Agents and Applications. Lecture Notes in Computer Science 1069: 179-190.

Huhns, M.N.; Stephens, L.M. 1999. Multiagent Systems and Societies of Agents, in Multiagent Systems: A Modern Approach to Distributed Artificial Intelligence. Gerhard Weiss, MIT Press, Cambridge, MA. 
Jenning, N. 2000. On agent-based software engineering. Artificial Inteligence 117:277-296

Koller, R. 2004. Un Enfoque de Sistemas Multi-Agentes para la Programación de la Producción en la Industria del Aserrío. Tesis de Magíster en Ciencia y Tecnología de la Madera. Universidad del Bio Bio, Concepción, Chile:23-51.

Maness, T.; Adams, D. 1991. The combined optimization of log bucking and sawing strategies. Wood and Fiber Science 23(2): 296-314.

Maness, T.C.; Scott E.N. 2002. Multiple Period Combined Optimization Approach to Forest Production Planning. Scandinavian Journal of Forest Research 17(5): 460- 471.

Mendoza, G.; Meimban, R.; Luppold, W; Araman, P. 1991. Combining simulation and optimization models for hardwood lumber production. SAP Nacional Convention, san Francisco, CA. 356-361.

Moyaux, T.; Chaib-draa, B. ; D'Amours, S. 2003. Multi-Agent Coordination Based on Tokens: Reduction of the Bullwhip Effect in a Forest Supply Chain. Proceedings of the $2^{\text {nd }}$ international joint conference on Autonomous Agents and MultiAgent Systems (AAMAS 2003).July: 14-18, Melbourne, Australia.

Moyaux, T. ; Chaib-draa, B. ; D'Amours, S. 2006. Supply Chain Management and Multiagent Systems: An Overview. Studies in Computational intelligence (SCI) 28: 1-27.

Ponce, M.; Contreras, M.; Vásquez, M. 2007. Exportación de madera aserrada de conífera chilena. Un análisis de su competitividad. Maderas. Ciencia y tecnología. 9(1):45-57.

Ribas-Vila, I.; Companys, P.R. 2006. Estado del arte de la Planificación Colaborativa en la Cadena de Suministro: Contexto Determinista e Incierto. DITS (2006/02). Working Paper del Departament d'Organització D'empreses de la Universitat Politècnica de Catalunya.

Santa-Eulalia, L.A.; Frayret, J.M.; D'Amours, S. 2007. Agent-based simulation for distributed supply chain planning: conceptual modeling, analysis and illustration. Interuniversity Research Centre on Enterprise Networks, Logistics and Transportation: CIRRELT 11: 1-27. Canadá, Quebec.

Singer, M.; Donoso, P. 2007. Internal supply chain management in the Chilean sawmill industry. International Journal of Operations y Production Management 27(5): 524-541.

Wessels, C. B.; Price, C.S.; Turner, P.; Dell, M.P. 2006. Integrating Harvesting and Sawmill operations using an optimized sawmill Production Planning System. Proceedings International Precision Forestry Symposium, 117-118. 